\title{
Utilization of Low Vision Aids in Buphthalmos
}

\author{
Partha Haradhan Chowdhury ${ }^{1 *}$ and Brinda Haren Shah ${ }^{2}$ \\ 1M.Optom, Associate Professor, Principal, Department of Optometry, Shree Satchandi \\ Jankalyan Samiti Netra Prasikshan Sansthan, Pauri, Affiliated to Uttarakhand State \\ Medical Faculty, Dehradun, India \\ ${ }^{2}$ M.Optom, Guest Lecturer, Department of Optometry, Shree Satchandi Jankalyan \\ Samiti Netra Prasikshan Sansthan, Pauri, Affiliated to Uttarakhand State Medical \\ Faculty, Dehradun, India
}

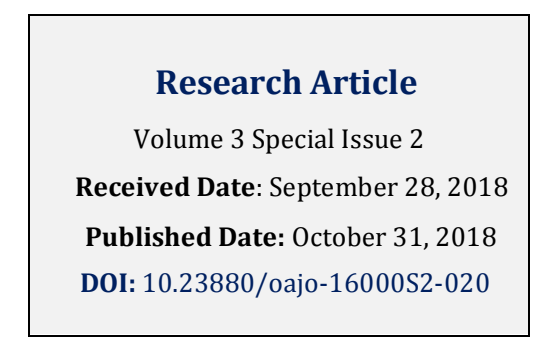

*Corresponding author: Partha Haradhan Chowdhury, M. Optom, Associate Professor, Principal, Department of Optometry, Shree Satchandi Jankalyan Samiti Netra Prasikshan Sansthan, Pauri, Affiliated to Uttarakhand State Medical Faculty, Dehradun, India, Email: optometrypublish@gmail.com

\section{Abstract}

Purpose: To analyse the utilization of the prescribed low vision device in the subjects of Buphthalmos.

Methods: Prospective, cross sectional and observational study was performed at low vision centers in Gujarat. All the visual functions were analysed after proper refractive correction. Low vision aids were prescribed and follow up was taken after 3 months to know the utilization of prescribed low vision devices. Data was analysed using SPSS software version 20 .

Results: 87 subjects were included in the study. Out of them, 61\% were males. It shows that spectacles of refractive correction (78\%) were mainly used by subjects for distance. For near, maximum CCTV (96\%) is used which is followed by stand magnifier (83\%). This can be taken into consideration while prescribing devices for Buphthalmos.

Conclusions: In cases of Buphthalmos, vision can be enhanced by illuminated stand magnifier or CCTV for near and spectacle correction for distance.

Keywords: Buphthalmos; Low vision aids

\section{Introduction}

Buphthalmos is a congenital glaucoma. It mainly occurs in the infantile stages of the subjects, so it is also known as Infantile Glaucoma. Here, aqueous outflow system is obstructed due to the encroachment of the cellular membrane at the angle of the anterior chamber and abnormal cleavage of the angle of the anterior chamber. Eyeball gets enlarged, sclera gets thinner, enlarged cornea, Tremulousness of the Iris, Flattened crystalline lens and rise in Intra ocular pressure occurs. That's why, whatever the retinal stimulation is needed for the brain that must be insufficient. Due to improper stimulation to the brain and damaged optic nerve head creates low vision to the subjects [1-3]. 


\section{Methodology}

A prospective, cross sectional and observational study was performed at low vision centers in Gujarat within the period of 3 years. All the subjects are enrolled with informed consent. Subjects having congenital buphthalmos and is considered in the low vision criteria were included in the study. Subjects who were below the low vision category and no pl vision were excluded from the study. Subjects having any other systemic disease associated were also excluded from the study. Visual acuity was assessed with various Log Mar charts. Contrast Sensitivity was assessed with Hiding Heidi charts and colour vision was assessed with PV 16 (Precision vision colour). All the functions were assessed after full refractive correction for distance and near. Optical, Non optical and electronic low vision aids were prescribed as per the need. Optical aids most commonly prescribed for distance was spectacles and telescopes (power $3 \mathrm{x}, 4 \mathrm{x}$ and $6 \mathrm{x}$ ) as required. For near, high add aspheric spectacles, reading bar magnifier and illuminated stand magnifier was prescribed. In, non optical devices, large print book and reading stand were prescribed for near. CCTV was prescribed as electronic magnifier. Follow up was taken after 3 months and analyzed which device was used maximum by the subjects. Data was analyzed using SPSS software version $20[4,5]$.

\section{Results}

A total of 87 subjects were included in the study. Figure 1 shows distribution of papers as per the age group. Figure 2 shows gender wise distribution of the subjects. Out of that, $61 \%$ of subjects were male and $39 \%$ were female. Figure 3 shows maximum usage of devices of subjects having Bupthalmos. It shows that spectacles of refractive correction (78\%) were mainly used by subjects for distance. For near, maximum cctv (96\%) is used which is followed by stand magnifier (83\%).

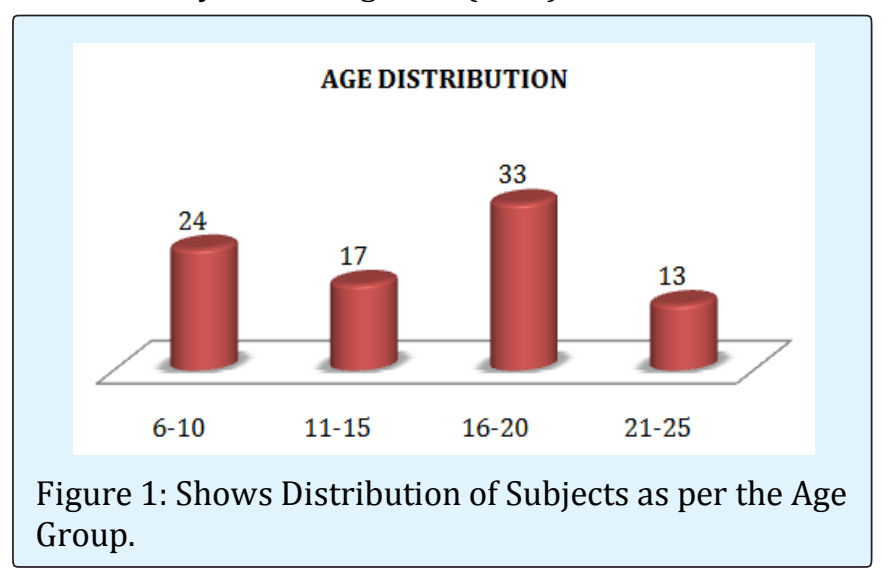

Partha Haradhan Chowdhury and Brinda Haren Shah. Utilization of Low Vision Aids in Buphthalmos. J Ophthalmol 2018, 3(S2): 000S2-020.
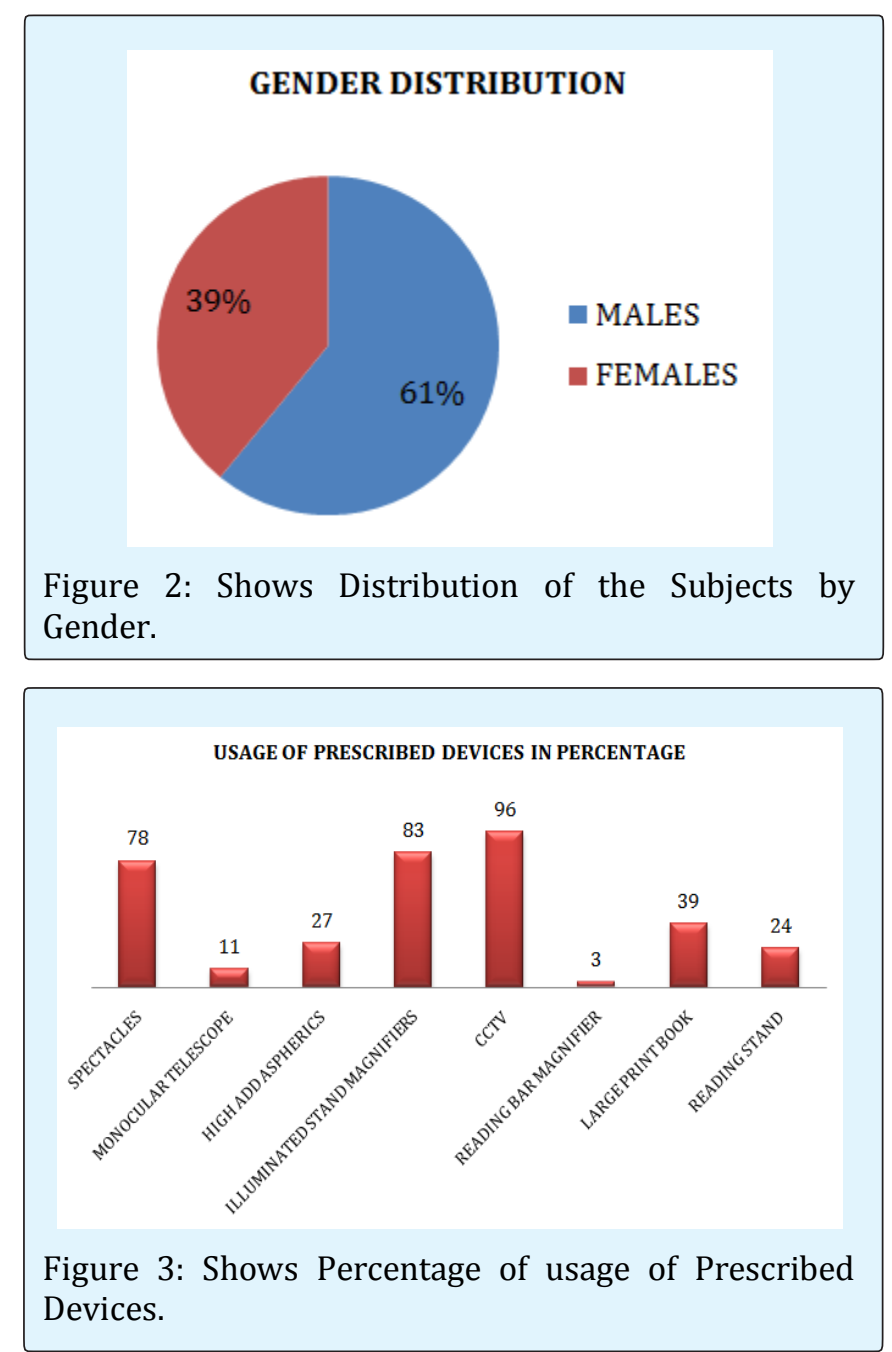

Chart 1 shows that maximum usage of devices used by the subject. This can be taken into consideration while prescribing devices for Buphthalmos [6,7].

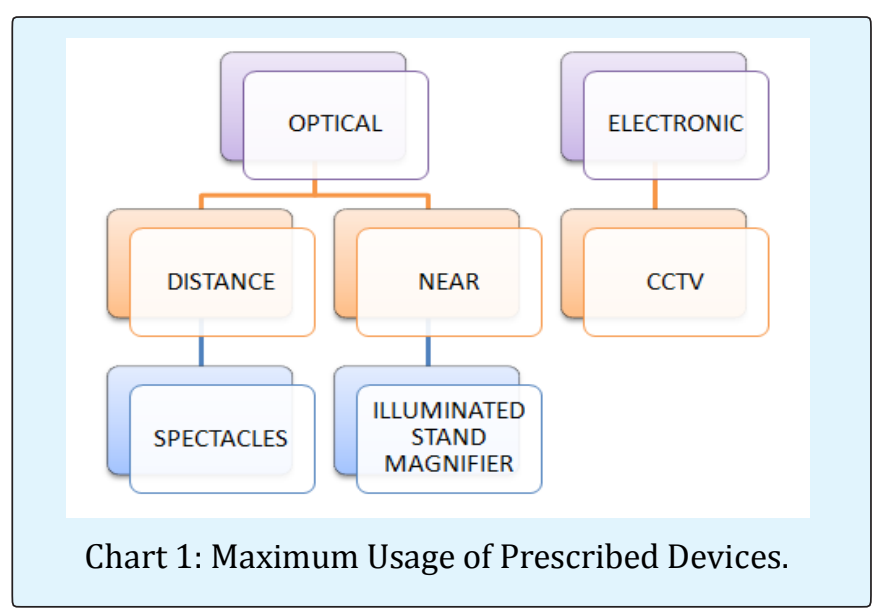

Copyright $($ Partha Haradhan Chowdhury and Brinda Haren Shah. 


\section{Open Access Journal of Ophthalmology}

\section{Discussion}

As per the study, it can be concluded that patients having Buphthalmos can be treated by the Low vision Aids usually for near purpose and significantly it is improved. In case of Buphthalmos, usually all the ocular structures are involved and damaged significantly, because outflow system is obstructed. By the low vision aids, it is treated and patient's response is positive significance. So, low visual aids must be provided in cases of Buphthalmos.

\section{Conclusions}

In cases of Buphthalmos, vision can be enhanced by illuminated stand magnifier or CCTV for near and spectacle correction for distance.

\section{References}

1. Richard L Brilliant (1999) Essentials of Low vision practice.

2. Bruce P Rosenthal, Roy Gordon Cole (1996) Functional Assessment of Low Vision.
3. Paul L Pease (2006) Borish's Clinical Refraction. William J Benjamin 2nd (Edn.).

4. Randall T Jose (1983) Understanding low vision.

5. Mitchell Scheiman, Maxine Scheiman, Steven Whittaker (2007) Low Vision Rehabilitation: A Practical Guide for Occupational Therapists.

6. Haddad MAO, Sei M, Braga AP, Sampaio MW, KaraJosé N (2000) Causes of visual impairment in childhood and adolescence: a retrospective study of 1917 cases. In: Stuen C, Arditi A, Horowitz A, Lang MA, Rosenthal B, Seidman K (Eds.), Vision Rehabilitation. Assessment, intervention and outcomes. New York: Swets \& Zeitlinger Publishers, pp: 371-375.

7. Simon JW, Buckely EG, Drack AV, Hutchinson AK, Plager AS, et al. (2003) Pediatric Ophthalmology and Strabismus. San Francisco: American Academy of Ophthalmology pp: 253-263.

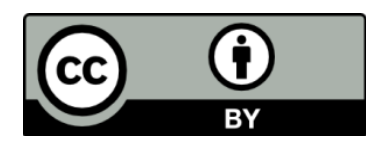

Partha Haradhan Chowdhury and Brinda Haren Shah. Utilization of Low Copyright@ Partha Haradhan Chowdhury and Brinda Haren Shah. 